\title{
NSAIDs and Breast Cancer Recurrence in a Prospective Cohort Study
}

\author{
Marilyn L. Kwan ${ }^{1}$, Laurel A. Habel ${ }^{1}$, Martha L. Slattery ${ }^{2}$, and Bette Caan ${ }^{1}$ \\ ${ }^{1}$ Division of Research, Kaiser Permanente, Oakland, CA 94612 \\ University of Utah, Salt Lake City, UT 84108
}

\section{Abstract}

Objective-We examined the association between NSAID use and breast cancer recurrence in a prospective cohort of 2292 early-stage breast cancer survivors diagnosed from 1997 to 2000 participating in the Life After Cancer Epidemiology (LACE) Study.

\begin{abstract}
Methods-From 2000 to 2002, mailed questionnaires were used to obtain information on aspirin, ibuprofen, and other NSAID use and subsequent breast cancer events. A total of 270 recurrences (local, regional, and distant disease and new primary breast cancers) were reported and verified by medical record review. Cox proportional hazard models were used to estimate rate ratios (RR) and 95\% confidence intervals (CI), adjusting for age at diagnosis, race, cancer stage, tamoxifen treatment, chemotherapy use, body mass index, and cyclooxygenase-2 (COX2) inhibitor use.
\end{abstract}

Results-Current, regular use (at least three days per week at time of questionnaire administration) of ibuprofen (RR, 0.56; 95\% CI, 0.32-0.98), but not aspirin (RR, 1.09; 95\% CI, $0.74-1.61$ ), was associated with a statistically significant decreased risk of breast cancer recurrence. The combination of ibuprofen and other non-aspirin NSAIDs such as naproxen and sulindac reflected a similar reduction in risk (RR, 0.56; 95\% CI, 0.33-0.95). No association was found for the non-NSAID analgesic acetaminophen.

Conclusion-Our findings provide support for an inverse association between current, regular ibuprofen use and breast cancer recurrence.

\section{Keywords}

Anti-Inflammatory Agents; Non-Steroidal; Breast Neoplasms; Recurrence; Chemoprevention

\section{Introduction}

Breast cancer survival rates have been increasing in the past decade with over two million survivors in the United States today [1]. As a result, it is becoming important to identify possible factors associated with breast cancer survivorship. Recently, some [2-8] but not all [9-11] observational studies have suggested that non-steroidal anti-inflammatory drugs (NSAIDs) such as aspirin may reduce the risk of breast cancer development, yet their association with breast cancer recurrence has not been explored.

To investigate the potential association between use of NSAIDs and risk of breast cancer recurrence, we analyzed data from the Life After Cancer Epidemiology (LACE) Study, a prospective cohort study of 2292 early stage breast cancer survivors that began in January 
2000. Using baseline data, NSAID use was analyzed according to overall current, regular use and if the use occurred before or after diagnosis.

\section{Materials and methods}

\section{Study cohort}

The LACE cohort has been described previously [12]. Briefly, the cohort consists of women diagnosed with invasive breast cancer from 1997 to 2000 recruited primarily from the Kaiser Permanente Northern California (KPNC) Cancer Registry (82\%) and the Utah Cancer Registry (12\%). Additionally, a subset of women who declined participation in the Women's Healthy Eating and Living (WHEL) Trial, a dietary intervention trial to prevent recurrence of breast cancer, was used as a third source of participants $(6 \%)$. Women were eligible if they were diagnosed from age 18-79 years with a first primary breast cancer (Stage I $\geq 1$ $\mathrm{cm}$, II, or IIIA) within 39 months of enrollment, had no history of other cancer within five years of enrollment, had completed cancer treatment (except for adjuvant hormonal therapy), and were recurrence-free at enrollment. Subsequently, after medical record review, any woman who had a recurrence, new breast primary, or death within three months of enrollment was deemed ineligible.

Between January 2000 and April 2002, 5656 women who were presumed to meet the LACE eligibility criteria were sent a recruitment package. Of these, $2614(46.2 \%)$ agreed to participate and completed the questionnaires. Subsequent medical record review to confirm eligibility resulted in 322 exclusions. Reasons for exclusions were diagnosed breast cancer recurrence/new primary or death within three months after enrollment (37\%), incorrect stage (34\%), other cancer within five years of enrollment (10\%), prior breast cancer (6\%), more than 39 months since diagnosis (6\%), incomplete demographic and medical data (3\%), still receiving treatment (2\%), and language difficulty (2\%). The remaining 2292 women constitute the LACE cohort. Women entered the cohort on average 1.90 years after diagnosis (range: 0.92 to 3.24 years).

\section{Ascertainment of NSAID use and other covariates}

All baseline data were obtained by mailed questionnaire. Women were asked "Are you CURRENTLY taking any of the following types of medications (at least 3 days per week)?" and "If Yes, when did you first start taking the medication?" Medications listed on the questionnaire included aspirin, ibuprofen, and acetaminophen. Use of other non-aspirin NSAIDs such as naproxen, sulindac, nabumetone, etodolac, meloxicam, and piroxicam were indicated in the "Other Medications" category. Hereafter, we refer to medication use of at least three days per week as "regular" use. Information on age, race/ethnicity, education, height and weight, smoking status, family history of breast cancer, and breast cancer treatment was also collected on the questionnaire. Post-diagnosis cyclooxygenase-2 (COX2) inhibitor use was assessed from the KPNC pharmacy prescription database such that individuals with more than a 100 day supply (at least two prescriptions) were considered users. Post-diagnosis use was determined a priori as the relevant exposure of interest in order to be concurrent with other non-selective NSAID use as collected from the questionnaire. Data on stage, nodal status, and tumor hormone receptor status were obtained from the Kaiser Permanente and Utah Cancer Registries and medical record review.

\section{Ascertainment of outcomes}

Subsequent health outcomes were ascertained semi-annually and after five years of followup annually by mailed questionnaire and verified by medical record. The average response rate to the mailed health status updates was $84 \%$. All non-respondents were called to complete a report by phone, increasing the response rate to $99 \%$. KPNC computerized 
mortality files were regularly searched or participant families were contacted for any cohort members not reached (1\%). For study subjects who were known to have died, copies of death certificates were obtained and cause of death recorded. Recurrences were defined as subsequent local, regional, or distant disease, new breast primary cancers in the contralateral breast, and deaths where the primary cause of death was from breast cancer and no recurrence was previously documented. For the small number of women in the latter group $(n=22)$, breast cancer recurrences were estimated to have occurred two years prior to death, following the methodology of Holmes et al. from the Nurses Health Study [13]. A total of 270 recurrences ( 36 local or regional, 199 distant, and 35 new breast cancers in the contralateral breast) were ascertained through December 12, 2006. Mean follow-up time from cohort entry until recurrence was 2.52 years.

\section{Data analysis}

To compare relevant characteristics of NSAID and non-NSAID users, Pearson chi-square tests were applied. Person-years were calculated for each participant, ranging from date of cohort entry to date of first confirmed cancer recurrence (as defined above), date of death (from causes other than breast cancer), drop-out date, or December 12, 2006, whichever occurred first. Rate ratios (RR) and 95\% confidence intervals (CI) for breast cancer recurrence were estimated by delayed entry Cox proportional hazard models, with months since diagnosis as the time scale [14]. The delayed entry model ensures that a woman who enrolled in the study $t$ years after her initial breast cancer diagnosis was not considered at risk for a possible recurrence prior to $t$ years by removing each woman from the risk set between the time of diagnosis and the time of cohort entry. Confidence intervals not overlapping with 1.00 or $p$ values $<0.05$ were considered to be statistically significant.

We examined two measures of aspirin, ibuprofen, and other NSAID use from questionnaire data: overall current, regular use (at least three days per week) at baseline questionnaire and if the use occurred before and/or after diagnosis. "No Use" of NSAIDs included only those individuals who reported never taking any NSAID. Duration was calculated as the total time period of use from date of initiation to date of questionnaire administration. The time period was then divided into no use (non-users and less than 3 month users), post-diagnosis use (greater than 3 month users after diagnosis only), and pre- and post-diagnosis use (greater than 3 month users both before and after diagnosis). Information was only obtained on duration and period of use among those who were current users at questionnaire administration such that past users who started and then stopped use (either pre- or postdiagnosis) were not asked to report this information. Therefore, pre-diagnosis use alone could not be determined.

To evaluate potential confounding by indication for NSAIDs, we also examined the association between current, regular use of acetaminophen and risk of recurrence. Acetaminophen is an analgesic and an antipyretic but has weak anti-inflammatory action and is not considered an NSAID. A priori confounders including age at diagnosis $(<50,50$ 59, 60-69, $\geq 70$ years), race (White, Black, Hispanic, Asian, Other), cancer stage (Stage I, IIA, IIB, IIIA), tamoxifen treatment (Never, Past, Current), chemotherapy use (Yes, No), body mass index $\left(<25,25-29,330 \mathrm{~kg} / \mathrm{m}^{2}\right)$, and post-diagnosis COX2 inhibitor use (Yes, No; treated as a time-dependent covariate) were statistically significant $(p<0.05)$ when added individually to the Cox models and thus were included in the final models as confounders. We also examined whether the associations between NSAID use and recurrence varied by menopausal status at diagnosis, BMI, tumor hormone receptor status, stage of initial breast cancer, and chemotherapy use by first generating strata-specific estimates and then including an interaction term in the model to test for statistical significance. 


\section{Results}

Most of the cohort members were white non-smokers who had no family history of breast cancer, regardless of NSAID use (Table 1). Women who used NSAIDs were more likely than non-users to be older, obese, and postmenopausal at diagnosis. NSAID users and nonusers were similar with respect to tamoxifen use, radiation therapy, stage of breast cancer, or hormone receptor status.

Current, regular use of ibuprofen was associated with a statistically significant decreased risk of breast cancer recurrence (RR, 0.56; 95\% CI, 0.32-0.98), which was not seen for aspirin (RR, 1.09; 95\% CI, 0.74-1.61) (Table 2). Although use of other non-aspirin NSAIDs such as naproxen, sulindac, nabumetone, etodolac, meloxicam, and piroxicam was not statistically significant (RR, $0.52 ; 95 \%$ CI, 0.13-2.13), the combination of these NSAIDs with ibuprofen was associated with a statistically significant decreased risk of breast cancer recurrence (RR, 0.56; 95\% CI, 0.33-0.95). Use of acetaminophen was associated with an increased, non-significant risk of recurrence (RR, 1.21; 95\% CI, 0.73-2.00). The associations between NSAID use and risk of recurrence did not appear to vary by menopausal status, BMI, tumor hormone receptor status, cancer stage, or chemotherapy use. Limiting the analysis to only distant recurrences produced similar results (not shown), although the association with ibuprofen use was no longer statistically significant (RR, 0.64; 95\% CI, 0.35-1.15).

The regular use of any NSAID (aspirin, ibuprofen, and other non-aspirin NSAID) during both the pre- and post-diagnosis period compared to no use was associated with a weak, non-statistically significant decreased risk of recurrence (RR, 0.87; 95\% CI, 0.56-1.33) (Table 2). However, the reduced risk appeared to be driven by ibuprofen (RR, $0.56 ; 95 \% \mathrm{CI}$, $0.28-1.15)$. Use of acetaminophen during the pre- and post-diagnosis period was not associated with recurrence (RR, 1.00; 95\% CI, 0.49-2.04). Interestingly, ibuprofen also showed a protective effect for post-diagnosis use only (RR, 0.55 ; 95\% CI, 0.22-1.34), but this observation was not seen for aspirin (RR, 1.23; 95\% CI, 0.72-2.11). Analyzing only distant recurrences yielded similar results (not shown).

\section{Discussion}

To our knowledge, this study is one of the first to specifically examine the relationship between use of NSAIDs and either breast cancer recurrence or survivorship. Among our cohort of early-stage breast cancer patients who had already survived on average the first two years following their diagnosis, we found that current, regular use of ibuprofen was associated with a statistically significant $44 \%$ reduction in risk of breast cancer recurrence. Post-diagnosis use of ibuprofen appeared to be most beneficial. Similar associations were observed for all non-aspirin NSAIDs. In contrast, aspirin use was not related to recurrence. It should be noted that since women did not enter the LACE study until they had completed chemotherapy and/or radiation treatment (on average two years post-diagnosis), our results cannot be generalized to recurrences that may have occurred in the initial post-diagnosis treatment period.

A variety of biological evidence supports the possible chemopreventive effects of NSAIDs. NSAIDs block the activity of COX enzymes 1 and 2 (COX1 and COX2), the rate-limiting step of prostaglandin biosynthesis. Under normal physiologic circumstances, COX1 is constitutively produced in most tissues while COX2 is induced by pro-inflammatory stimuli [15]. Inhibition of COX2 by NSAIDs might induce apoptosis and hinder angiogenesis, both of which are involved in carcinogenesis [16]. Some mechanistic studies suggest that nonaspirin NSAIDs have greater effects on COX2 inhibition than aspirin [17, 18]. Since 
carcinogenesis is believed to be a multi-step process (initiation, promotion, and progression), and diagnosis is an arbitrary time point along this continuum [19], use of NSAIDs may be associated with cancer progression, as well as cancer development. COX2 overexpression was detected in human breast carcinomas [20, 21] and linked to markers of angiogenesis and apoptosis [22]. More importantly, COX2 overexpression was associated with unfavorable distant disease-free survival among a large sample size of 1576 invasive breast cancers [23]. In our cohort, only 61 women were considered COX2 users, precluding a full analysis of the association between $\mathrm{COX} 2$ inhibitor use and risk of breast cancer recurrence, yet COX2 use was treated as a potential confounder in this current analysis of non-selective NSAIDs.

To our knowledge, only one study has assessed the possible role of NSAID use on breast cancer survival [24]. In this study of 591 postmenopausal women, reduced risks of breast cancer mortality $(\mathrm{HR}=0.64 ; 95 \% \mathrm{CI}: 0.39-1.05)$ and all-cause mortality $(\mathrm{HR}=0.57 ; 95 \%$ CI: 0.40-0.81) were reported for aspirin and non-aspirin NSAID use. Although the breast cancer endpoints differ slightly, our results are in agreement with those of this smaller study indicating a protective effect by non-aspirin NSAIDs.

Strengths of the LACE study include being one of the few existing, relatively large cohorts of early-stage breast cancer survivors with information on post-diagnosis behaviors, and one of the first studies to examine the potential association between NSAID use and breast cancer recurrence. A limitation of this study is the lack of questionnaire data on frequency or dose of NSAID use, yet intake of NSAIDs at least three days per week was specifically asked. In addition, only current users of NSAIDs were required to provide information on dates of use, and therefore we were unable to identify women who were only past users. Fortunately, this misclassification would most likely be non-differential such that our risk estimates would be attenuated towards a null association. Furthermore, use of some NSAIDs (e.g. naproxen) was not explicitly queried on the questionnaire, increasing the possibility that this information was incompletely recorded. Finally, our follow-up period for the cohort was an average of 5.00 years, which might not be enough time to accumulate sufficient outcomes to detect a true association. Given the exposure prevalence of $24 \%$ and verified recurrences of 270 in the present cohort, we had the power to detect relative risks of approximately 0.65 or lower. Longer follow-up may produce more definitive results.

In conclusion, our results provide support for an inverse association between current, regular use of ibuprofen and risk of breast cancer recurrence and are suggestive of an association with other non-aspirin NSAIDs. Other studies are needed to confirm our findings and to further explore NSAID type, duration, and dose. We will continue to investigate these issues in the LACE cohort using follow-up data in the permanent survival period (five to seven years post-diagnosis).

\section{Acknowledgments}

This study was funded by the National Cancer Institute (R01 CA80027) and by the Utah Cancer Registry (N01 PC67000), with additional support from the State of Utah Department of Health. We thank Charles Quesenberry, Ph.D. for his biostatistical support and Robert Reynolds, M.P.H. for his programming support. We thank all LACE Study staff and participants.

Financial Support: National Cancer Institute (R01 CA80027 and N01 PC67000)

\section{References}

1. American Cancer Society. 2006. http://www.cancer.org 
2. Harris RE, Chlebowski RT, Jackson RD, et al. Breast cancer and nonsteroidal anti-inflammatory drugs: prospective results from the Women's Health Initiative. Cancer Res. 2003; 63(18):6096-101. [PubMed: 14522941]

3. Khuder SA, Mutgi AB. Breast cancer and NSAID use: a meta-analysis. Br J Cancer. 2001; 84(9): 1188-92. [PubMed: 11336469]

4. Moorman PG, Grubber JM, Millikan RC, Newman B. Association between non-steroidal antiinflammatory drugs (NSAIDs) and invasive breast cancer and carcinoma in situ of the breast. Cancer Causes Control. 2003; 14(10):915-22. [PubMed: 14750530]

5. Swede H, Mirand AL, Menezes RJ, Moysich KB. Association of regular aspirin use and breast cancer risk. Oncology. 2005; 68(1):40-7. [PubMed: 15802928]

6. Terry MB, Gammon MD, Zhang FF, et al. Association of frequency and duration of aspirin use and hormone receptor status with breast cancer risk. Jama. 2004; 291(20):2433-40. [PubMed: 15161893]

7. Zhang Y, Coogan PF, Palmer JR, Strom BL, Rosenberg L. Use of nonsteroidal antiinflammatory drugs and risk of breast cancer: the Case-Control Surveillance Study revisited. Am J Epidemiol. 2005; 162(2):165-70. [PubMed: 15972932]

8. Gonzalez-Perez A, Garcia Rodriguez LA, Lopez-Ridaura R. Effects of non-steroidal antiinflammatory drugs on cancer sites other than the colon and rectum: a meta-analysis. BMC Cancer. 2003; 3:28. [PubMed: 14588079]

9. Cook NR, Lee IM, Gaziano JM, et al. Low-dose aspirin in the primary prevention of cancer: the Women's Health Study: a randomized controlled trial. Jama. 2005; 294(1):47-55. [PubMed: 15998890]

10. Jacobs EJ, Thun MJ, Connell CJ, et al. Aspirin and other nonsteroidal anti-inflammatory drugs and breast cancer incidence in a large U.S. cohort. Cancer Epidemiol Biomarkers Prev. 2005; 14(1): 261-4. [PubMed: 15668504]

11. Marshall SF, Bernstein L, Anton-Culver H, et al. Nonsteroidal anti-inflammatory drug use and breast cancer risk by stage and hormone receptor status. J Natl Cancer Inst. 2005; 97(11):805-12. [PubMed: 15928301]

12. Caan B, Sternfeld B, Gunderson E, Coates A, Quesenberry C, Slattery ML. Life After Cancer Epidemiology (LACE) Study: a cohort of early stage breast cancer survivors (United States). Cancer Causes Control. 2005; 16(5):545-56. [PubMed: 15986109]

13. Holmes MD, Chen WY, Feskanich D, Kroenke CH, Colditz GA. Physical activity and survival after breast cancer diagnosis. Jama. 2005; 293(20):2479-86. [PubMed: 15914748]

14. Hosmer, DW.; Lemeshow, S. Applied Survival Analysis: Regression Modeling of Time to Event Data. John Wiley \& Sons, Inc; New York: 1999.

15. Ulrich CM, Bigler J, Potter JD. Non-steroidal anti-inflammatory drugs for cancer prevention: promise, perils and pharmacogenetics. Nat Rev Cancer. 2006; 6(2):130-40. [PubMed: 16491072]

16. Meric JB, Rottey S, Olaussen K, et al. Cyclooxygenase-2 as a target for anticancer drug development. Crit Rev Oncol Hematol. 2006; 59(1):51-64. [PubMed: 16531064]

17. Alshafie GA, Harris RE, Robertson FM, Parrett ML, Ross M, Abou-Issa H. Comparative chemopreventive activity of ibuprofen and $\mathrm{N}$-(4-hydroxyphenyl) retinamide against the development and growth of rat mammary adenocarcinomas. Anticancer Res. 1999; 19(4B):30316. [PubMed: 10652588]

18. Robertson FM, Parrett ML, Joarder FS, et al. Ibuprofen-induced inhibition of cyclooxygenase isoform gene expression and regression of rat mammary carcinomas. Cancer Lett. 1998; 122(1-2): 165-75. [PubMed: 9464506]

19. Arun B, Hortobagyi GN. Progress in breast cancer chemoprevention. Endocr Relat Cancer. 2002; 9(1):15-32. [PubMed: 11914180]

20. Hwang D, Scollard D, Byrne J, Levine E. Expression of cyclooxygenase-1 and cyclooxygenase-2 in human breast cancer. J Natl Cancer Inst. 1998; 90(6):455-60. [PubMed: 9521170]

21. Parrett M, Harris R, Joarder F, Ross M, Clausen K, Robertson F. Cyclooxygenase-2 gene expression in human breast cancer. Int J Oncol. 1997; 10:503-8. [PubMed: 21533404] 
22. Costa C, Soares R, Reis-Filho JS, Leitao D, Amendoeira I, Schmitt FC. Cyclo-oxygenase 2 expression is associated with angiogenesis and lymph node metastasis in human breast cancer. $\mathrm{J}$ Clin Pathol. 2002; 55(6):429-34. [PubMed: 12037025]

23. Ristimaki A, Sivula A, Lundin J, et al. Prognostic significance of elevated cyclooxygenase-2 expression in breast cancer. Cancer Res. 2002; 62(3):632-5. [PubMed: 11830510]

24. Blair CK, Sweeney C, Anderson KE, Folsom AR. NSAID use and survival after breast cancer diagnosis in post-menopausal women. Breast Cancer Res Treat. 2006 
Table 1

Demographic characteristics and breast cancer risk factors by current, regular NSAID use (at least 3 days per week) in the LACE Study ( $n=2292), 2000-2006$

\begin{tabular}{|c|c|c|c|}
\hline & Yes NSAID Use $^{a}$ No. (\%) & No NSAID Use No. (\%) & $p$ value $^{b}$ \\
\hline Age at diagnosis (years) & & & $<0.001$ \\
\hline$<50$ & 77 (13.9) & $483(27.8)$ & \\
\hline $50-59$ & $152(27.4)$ & $551(31.7)$ & \\
\hline $60-69$ & $190(34.2)$ & $447(25.7)$ & \\
\hline$\geq 70$ & $136(24.5)$ & $256(14.8)$ & \\
\hline Mean \pm Standard Deviation & $61.8 \pm 10.2$ & $57.2 \pm 11.0$ & \\
\hline Race/ethnicity & & & $<0.01$ \\
\hline White & $468(84.5)$ & $1373(79.3)$ & \\
\hline Black & $27(4.9)$ & $86(5.0)$ & \\
\hline Hispanic & $27(4.9)$ & $116(6.7)$ & \\
\hline Asian & $18(3.2)$ & $113(6.5)$ & \\
\hline Other & $14(2.5)$ & $44(2.5)$ & \\
\hline Unknown & 1 & 5 & \\
\hline Education & & & 0.48 \\
\hline$\leq$ HS diploma/GED & $146(26.5)$ & $477(27.5)$ & \\
\hline Some college & $237(42.9)$ & $614(35.5)$ & \\
\hline$\geq$ College degree & $169(30.6)$ & $640(37.0)$ & \\
\hline Unknown & 3 & 6 & \\
\hline Smoking history & & & 0.61 \\
\hline Never & $278(50.2)$ & $931(54.0)$ & \\
\hline Past & $243(43.9)$ & $653(37.8)$ & \\
\hline Current & $33(5.9)$ & $141(8.2)$ & \\
\hline Unknown & 1 & 12 & \\
\hline Body mass index $(\mathrm{BMI})$ at prediagnosis $\left(\mathrm{kg} / \mathrm{m}^{2}\right)$ & & & $<0.001$ \\
\hline$<25$ & $220(39.6)$ & $839(48.3)$ & \\
\hline $25-29$ & $178(32.1)$ & $507(29.2)$ & \\
\hline$\geq 30$ & $157(28.3)$ & $391(22.5)$ & \\
\hline Mean \pm Standard Deviation & $27.7 \pm 6.1$ & $26.6 \pm 5.7$ & \\
\hline Menopausal status at diagnosis ${ }^{c}$ & & & $<0.001$ \\
\hline Premenopausal & $71(14.7)$ & $447(29.9)$ & \\
\hline Postmenopausal & $413(85.3)$ & $1047(70.1)$ & \\
\hline Unknown & 71 & 243 & \\
\hline Family history of breast cancer & & & 0.97 \\
\hline No & $441(79.5)$ & $1374(79.4)$ & \\
\hline Yes & $114(20.5)$ & $357(20.6)$ & \\
\hline Unknown & 0 & 6 & \\
\hline Radiation therapy & & & 0.65 \\
\hline No & $204(37.6)$ & $619(36.5)$ & \\
\hline
\end{tabular}




\begin{tabular}{|c|c|c|c|}
\hline & Yes NSAID Use ${ }^{a}$ No. (\%) & No NSAID Use No. (\%) & $p$ value \\
\hline Yes & $339(62.4)$ & $1077(63.5)$ & \\
\hline Unknown & 12 & 41 & \\
\hline Chemotherapy & & & $<0.001$ \\
\hline No & $283(51.4)$ & $693(40.3)$ & \\
\hline Yes & $268(48.6)$ & $1025(59.7)$ & \\
\hline Unknown & 4 & 19 & \\
\hline Tamoxifen & & & 0.56 \\
\hline Never & $133(24.2)$ & $364(21.4)$ & \\
\hline Past & $22(4.0)$ & $125(7.3)$ & \\
\hline Current & $394(71.8)$ & $1215(71.3)$ & \\
\hline Unknown & 6 & 33 & \\
\hline Stage of breast cancer & & & 0.33 \\
\hline Stage I & $269(48.6)$ & $798(46.2)$ & \\
\hline Stage IIA & $186(33.6)$ & $589(34.1)$ & \\
\hline Stage IIB & $81(14.6)$ & $289(16.7)$ & \\
\hline Stage IIIA & $18(3.2)$ & $52(3.0)$ & \\
\hline Unknown & 1 & 9 & \\
\hline Nodal status & & & $<0.01$ \\
\hline Negative & $356(68.7)$ & 1009 (61.6) & \\
\hline Positive & $162(31.3)$ & $630(38.4)$ & \\
\hline Unknown & 37 & 98 & \\
\hline Estrogen/Progesterone receptor status & & & 0.68 \\
\hline NegNeg & $79(16.4)$ & $234(15.1)$ & \\
\hline NegPos & $9(1.9)$ & $27(1.8)$ & \\
\hline PosNeg & $57(11.8)$ & $211(13.6)$ & \\
\hline PosPos & $336(69.9)$ & $1075(69.5)$ & \\
\hline Unknown & 74 & 190 & \\
\hline $\mathrm{C} 0 \mathrm{X} 2$ inhibitor use ${ }^{d}$ & & & 0.49 \\
\hline No Use & $443(95.7)$ & $1438(96.4)$ & \\
\hline Ever Use & $20(4.3)$ & $54(3.6)$ & \\
\hline Unknown & 92 & 245 & \\
\hline Total & 555 & 1737 & 2292 \\
\hline
\end{tabular}

${ }^{a}$ NSAID use includes current use of aspirin, ibuprofen, and other non-aspirin NSAIDs such as naproxen, sulindac, nabumetone, etodolac, meloxicam, and piroxicam.

$b_{\text {From Pearson chi-square test. }}$

${ }^{c}$ Premenopausal at diagnosis includes less than 60 years old at diagnosis, had period within three months prior to diagnosis, and not on estrogen HT prior to diagnosis; Postmenopausal at diagnosis includes the following four scenarios: 1) greater than or equal to 60 years old at diagnosis or periods stopped 12 months or more before diagnosis (excludes women who had a hysterectomy only before diagnosis, with last period within seven months of hysterectomy), 2) less than 60 years old at diagnosis and had oophorectomy and hysterectomy any time before diagnosis, with periods stopping at the same time as surgery, 3) less than 60 years old at diagnosis and had oophorectomy without hysterectomy before diagnosis, with HT beginning around oophorectomy date, 4) less than 60 years old at diagnosis and had oophorectomy without hysterectomy before diagnosis date, with periods stopping at same time as oophorectomy and having no HT before diagnosis; Unknown includes any scenario not covered above. 
$d_{\text {"No Use" includes }} \leq 00$ days supply and "Ever Use" includes $>100$ days supply obtained from KPNC pharmacy prescription database. 
Table 2

Relative risks of breast cancer recurrence and current, regular NSAID use (at least 3 days per week) at time of questionnaire administration $(n=2292)$ in the LACE Study, 2000-2006

\begin{tabular}{|c|c|c|c|c|}
\hline Type of NSAID $^{a}$ & Recurrences $^{b} n(\%)$ & Non-recurrences $n(\%)$ & $\begin{array}{l}\text { Unadjusted RR }(95 \% \\
\text { CI })^{c}\end{array}$ & $\begin{array}{c}\text { Adjusted RR }(95 \% \\
\text { CI }) \\
c, d\end{array}$ \\
\hline \multicolumn{5}{|l|}{ Aspirin } \\
\hline No Use & $209(83.6)$ & $1528(82.9)$ & 1.00 (reference) & 1.00 (reference) \\
\hline Ever Use & $41(16.4)$ & $314(17.1)$ & $0.94(0.67-1.32)$ & $1.09(0.74-1.61)$ \\
\hline No Use & $209(83.6)$ & $1528(82.9)$ & 1.00 (reference) & 1.00 (reference) \\
\hline Post-diagnosis Use & $18(7.2)$ & $130(7.1)$ & $0.98(0.60-1.61)$ & $1.23(0.72-2.11)$ \\
\hline Pre- and Post-diagnosis Use & $23(9.2)$ & $184(10.0)$ & $0.91(0.59-1.41)$ & $0.99(0.60-1.64)$ \\
\hline Total duration of use (years) & \multicolumn{2}{|c|}{ Median: 2.82} & \multicolumn{2}{|c|}{ Mean: 8.24} \\
\hline \multicolumn{5}{|l|}{ Ibuprofen } \\
\hline No Use & $209(90.9)$ & $1528(87.9)$ & 1.00 (reference) & 1.00 (reference) \\
\hline Ever Use & $21(9.1)$ & $211(12.1)$ & $0.71(0.45-1.12)$ & $0.56(0.32-0.98)$ \\
\hline No Use & $209(90.9)$ & $1528(87.9)$ & 1.00 (reference) & 1.00 (reference) \\
\hline Post-diagnosis Use & $9(3.9)$ & $87(5.0)$ & $0.67(0.33-1.37)$ & $0.55(0.22-1.34)$ \\
\hline Pre- and Post-diagnosis Use & $12(5.2)$ & $124(7.1)$ & $0.73(0.41-1.31)$ & $0.56(0.28-1.15)$ \\
\hline Total duration of use (years) & \multicolumn{2}{|c|}{ Median: 2.98} & \multicolumn{2}{|c|}{ Mean: 6.34} \\
\hline \multicolumn{5}{|l|}{ Other Non-aspirin NSAID ${ }^{e}$} \\
\hline No Use & 209 (98.6) & $1528(98.2)$ & 1.00 (reference) & 1.00 (reference) \\
\hline Ever Use & $3(1.4)$ & $28(1.8)$ & $0.53(0.13-2.12)$ & $0.52(0.13-2.13)$ \\
\hline No Use & 209 (98.6) & $1528(98.2)$ & 1.00 (reference) & 1.00 (reference) \\
\hline Post-diagnosis Use & $3(1.4)$ & $15(1.0)$ & $0.93(0.23-3.75)$ & $0.78(0.19-3.18)$ \\
\hline Pre- and Post-diagnosis Use & $0(0)$ & $13(0.8)$ & not calculable & not calculable \\
\hline Total duration of use (years) & \multicolumn{2}{|c|}{ Median: 0.99} & \multicolumn{2}{|c|}{ Mean: 3.83} \\
\hline \multicolumn{5}{|l|}{ Any NSAID ${ }^{f}$} \\
\hline No Use & $209(77.4)$ & $1528(75.6)$ & 1.00 (reference) & 1.00 (reference) \\
\hline Ever Use & $61(22.6)$ & $494(24.4)$ & $0.87(0.65-1.17)$ & $0.90(0.65-1.26)$ \\
\hline No Use & 209 (77.4) & $1528(75.6)$ & 1.00 (reference) & 1.00 (reference) \\
\hline Post-diagnosis Use & $28(10.4)$ & $220(10.9)$ & $0.87(0.58-1.31)$ & $0.94(0.60-1.48)$ \\
\hline Pre- and Post-diagnosis Use & $33(12.2)$ & $274(13.5)$ & $0.88(0.61-1.28)$ & $0.87(0.56-1.33)$ \\
\hline Total duration of use (years) & \multicolumn{2}{|c|}{ Median: 2.80} & \multicolumn{2}{|c|}{ Mean: 6.89} \\
\hline \multicolumn{5}{|l|}{ Acetaminophen } \\
\hline No Use & $250(92.6)$ & $1876(92.8)$ & 1.00 (reference) & 1.00 (reference) \\
\hline Ever Use & $20(7.4)$ & $146(7.2)$ & $1.06(0.67-1.68)$ & $1.21(0.73-2.00)$ \\
\hline No Use & $250(92.6)$ & $1876(92.8)$ & 1.00 (reference) & 1.00 (reference) \\
\hline Post-diagnosis Use & $10(3.7)$ & $64(3.2)$ & $1.19(0.63-2.24)$ & $1.49(0.76-2.93)$ \\
\hline Pre- and Post-diagnosis Use & $10(3.7)$ & $82(4.0)$ & $0.96(0.51-1.81)$ & $1.00(0.49-2.04)$ \\
\hline Total duration of use (years) & \multicolumn{2}{|c|}{ Median: 2.50} & \multicolumn{2}{|c|}{ Mean: 8.65} \\
\hline
\end{tabular}




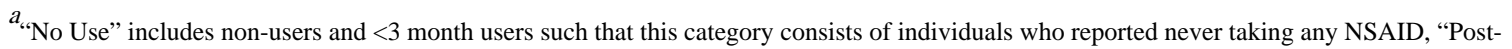
diagnosis Use" includes greater than 3 month users after diagnosis only, and "Pre- and Post-diagnosis Use" includes greater than 3 month users both before and after diagnosis.

$b_{270}$ total breast cancer recurrences (36 local or regional, 199 distant, and 35 new breast cancers in the contralateral breast).

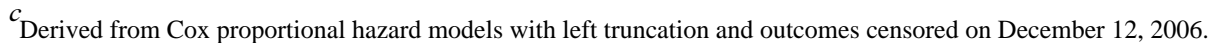
${ }^{d}$ Adjusted for age at diagnosis, race, stage of breast cancer, tamoxifen treatment, body mass index, chemotherapy use, and post-diagnosis COX2 inhibitor use (time-dependent covariate) as delineated in Table 1.

Other NSAID includes naproxen, sulindac, nabumetone, etodolac, meloxicam, and piroxicam.

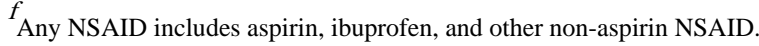

